\title{
Mejoras tecnológicas en el proceso de inyección de carbón pulverizado en el horno alto ${ }^{(\bullet)}$
}

\author{
A. $\operatorname{Babich}^{(*)}$, S. Yaroshevskii ${ }^{(*)}$, L. García $^{(* *)}$, A. Formoso $^{(* * *)}$, A. Cores $^{(* * *)}$, \\ A. Isidro ${ }^{(* * *)}$ y S. Ferreira $(* * *)$
}

Resumen Se estudia la tecnología de operación del horno alto con inyección de carbón pulverizado (ICP) con el fin de sustituir el máximo posible de coque siderúrgico en la carga y se analizan aspectos de la combustión del carbón pulverizado (CP) y los métodos y dispositivos elaborados para intensificar su combustión en la zona de toberas del horno alto. Se ofrecen recomendaciones para optimizar la molienda del carbón, se estudia la problemática de la distribución del CP a lo largo de la periferia del horno y se describe una tecnología elaborada para compensar las perturbaciones producidas por la inyección de $\mathrm{CP}$ en el horno alto sobre diferentes parámetros, tales como grado de reducción directa, intercambio térmico y otras.

Palabras clave: Carbón pulverizado. Inyección de carbón pulverizado. Toberas. Horno alto.

\section{Technological improvements in the pulverized coal injection process in the blast furnace}

\begin{abstract}
Blast furnace operation technology with pulverized coal injection (PCI) has been carried out in order to replace the maximum amount of metallurgical coke in the burden. PC burning has been studied and methods and designs for the intensification of its combustion have been developed within the raceway. Recommendations for optimizing coal grinding have been developed. Problems of PC distribution inside the circumference of the furnace were investigated. Compensating technology for changes in reduction, heat exchange and other processes under PCI have been developed.
\end{abstract}

Keywords: Pulverized coal. Pulverized coal injection. Tuyeres. Blast furnace.

\section{INTRODUCCIÓN}

La idea de inyectar combustible por tobera al horno alto ya fue propuesta en 1831 por John Samuel Dawes (1). Sin embargo, el estudio de este proceso y la preparación de equipamiento y tecnología de fabricación de arrabio con utilización de $\mathrm{CP}$ se inició a comienzos de los años 60 del presente siglo en China, Ucrania y en los EE.UU. (2-6). Las primeras instalaciones industriales de ICP se

$(\bullet \quad$ Trabajo recibido el día 1 de marzo de 1996

(*) Universidad Estatal Tecnológica de Donetsk. Avda. Arteoma, 58. 340066 Donetsk (Ucrania).

(**) Unión de Empresas de Recuperación de Materias Primas. 1 ra. Avda. 1604. 11300 Ciudad de La Habana (Cuba).

(***) Centro Nacional de Investigaciones Metalúrgicas (CSIC). Avda. de Gregorio del Amo, 8. 28040-Madrid (España). introdujeron en esa época en las fábricas de Armco Steel, en Askland (EE.UU.), y en Shoudu, en Pekín (República Popular China). En Europa, la primera instalación industrial para preparación e inyección de CP se construyó en Donetsk (Ucrania) y se puso en explotación en 1980 (7). En 1981 entró en funcionamiento la primera instalación de este tipo en Japón (8).

Debido al considerable crecimiento de los precios del gas natural y del petróleo, en comparación con el carbón, en los últimos 15 años, el CP ha pasado a ser el principal combustible auxiliar, empleado como sustituto del coque en la operación del horno alto. En cerca de 20 países operan aproximadamente 100 instalaciones con esta tecnología y, actualmente, se proyectan y construyen numerosas plantas. Con la utilización de ICP operan actualmente unos 40 hornos altos en países de Europa Occidental, 27 de los 29 hornos en operación en 
Japón, el 75 \% de los hornos de la República Popular China, así como una serie de hornos en EE.UU., Corea del Sur, Taiwan y en otros países. Los niveles de inyección en algunos hornos altos (HA-1, de Kokogawa Works (Japón), HA-6, de Hoogovens (Holanda) y Queen Victoria, de British Steel, en Scunthorpe (Reino Unido)), en determinados períodos han alcanzado los $200 \mathrm{~kg}$ por tonelada de arrabio (kg/t a) (9).

Sin embargo, al incrementar los niveles de inyección de $\mathrm{CP}$ con el fin de reducir al máximo el consumo de coque y la amortización de las costosas instalaciones de preparación e inyección del $\mathrm{CP}$, se originan una serie de perturbaciones en la marcha del horno. Los factores principales que dificultan la inyección de cantidades elevadas de CP son:

- El problema de garantizar un alto grado de gasificación de las partículas de carbón en la zona de toberas.

- La distribución uniforme del carbón a lo largo de la periferia del horno.

- La compensación de las perturbaciones que surgen en la operación del horno.

- Una marcha estable del horno cuando opera con cantidades pequeñas de coque en la carga.

En el presente trabajo se estudian las posibles soluciones a estos problemas.

\section{GASIFICACIÓN DEL CP EN LA ZONA DE TOBERAS}

\subsection{Teoría de la combustión del combustible sólido}

El combustible sólido está formado por un conjunto complejo de compuestos orgánicos y sustancias minerales. La combustión del carbón contenido en el combustible sólido es un proceso heterogéneo, que se produce en la superficie del sólido y en la fase gaseosa. El proceso está regido por la cinética de la combustión, tanto en la superficie como en el interior de las partículas, y por la difusión del oxígeno y de los productos de la combustión. La velocidad total del proceso corresponde a la velocidad de la etapa más lenta, y debido a ello, la etapa controlante del proceso puede ser la difusión, la cinética o una intermedia. El carácter del proceso de combustión depende de la magnitud de la relación:

$$
K_{1} \cdot \delta_{1} /(N u \cdot D)
$$

donde

$K_{1}=$ Constante de la velocidad de combustión, $\mathrm{m} / \mathrm{s}$;

$\delta_{1}=$ Tamaño máximo de las partículas, $\mathrm{m}$;

$N u=$ Número de Nusselt;
$D=$ Coeficiente de difusión del oxígeno en el gas en la zona de combustión, $\mathrm{m}^{2} / \mathrm{s}$.

Si el valor de la relación [1] es $<1$, el proceso está controlado por la difusión; en caso contrario, por la cinética. También es un factor muy importante el tamaño de partícula del combustible.

En la combustión del combustible sólido ejercen influencia los contenidos de sustancias volátiles y de ceniza del propio combustible. Durante la inyección del CP en la zona de toberas, el grado de combustión, a diferencia de lo que sucede en el proceso en lecho estático, se determina por la siguiente ecuación:

$G=1-K \int_{\left(\delta_{\mathrm{oi}}\right)_{\tau}} \delta_{01} \frac{\left|\mathrm{d} R_{\mathrm{oi}}\right|}{\mathrm{d} \delta_{\mathrm{oi}}}\left(\frac{\delta_{\mathrm{i}}}{\delta_{\mathrm{oi}}}\right)^{3} \mathrm{~d} \delta_{\mathrm{oi}}$

donde

$G=$ Grado de combustión;

$K=$ Contenido relativo de carbono residual en la masa del combustible, $\mathrm{kg} / \mathrm{kg}$;

$\delta_{\text {oi }}$ y $\delta_{\mathrm{i}}=$ Tamaños inicial y transitorio de las partículas de combustible en la fracción $i$, respectivamente, $\mathrm{m}$;

$\delta_{\mathrm{o} 1}=$ Tamaño inicial de partícula de la fracción mayor, $\mathrm{m}$;

$\delta\left({ }_{\mathrm{oi}}\right) \tau=$ Tamaño inicial de la partícula completamente quemada, en el tiempo $\tau$, $\mathrm{m}$;

$R_{\mathrm{oi}}=$ Contenido relativo (residuo en la malla) de las partículas con tamaño igual o superior a $\delta_{\mathrm{oi}}$ en el polvo inicial.

\subsection{Estudios teóricos y experimentales de la combustión del $\mathbf{C P}$}

Para los cálculos, se utiliza el método de la determinación del grado de combustión del CP, elaborado para hogares de combustión (10) y adaptado a las condiciones de combustión de llama polidispersa en la zona de toberas del horno alto (11).

Los cálculos se realizan para las condiciones de operación del horno con viento atmosférico y enriquecido con oxígeno para una temperatura adiabática de $1.900-2.200{ }^{\circ} \mathrm{C}$. La tasa de inyección de $\mathrm{CP}$ varía desde 0 hasta $240 \mathrm{~kg} / \mathrm{t}$ a. El CP procede de hullas magras, cuyo tamaño máximo de partícula varia desde 0 hasta $1.000 \mu \mathrm{m}$.

Los resultados de los cálculos muestran que, en todos los intervalos de temperatura estudiados en la combustión de CP con un tamaño máximo de intervalo de partículas superior a 50-100 $\mu \mathrm{m}$, la relación entre el coeficiente de difusión del oxígeno en el gas en la zona de combustión y la constante de la velocidad de reacción es tal que la magnitud [1] es $<1$.

El CP empleado en la práctica tiene un tamaño máximo de partícula de 200-400 $\mu \mathrm{m}$ (con una 
media ponderada de 40-80 $\mu \mathrm{m}$ ). Por tanto, se puede afirmar que la etapa controlante de la combustión del CP en horno alto es la difusión.

$\mathrm{El}$ grado de combustión del $\mathrm{CP}$ se reduce considerablemente con el incremento de su tasa de inyección. Así pues, para altas tasas de CP, es necesario tomar medidas especiales para intensificar su combustión.

Las investigaciones de laboratorio se realizan en una instalación que simula las condiciones de combustión del CP en la zona de toberas (Fig. 1). Se realizan ensayos para estudiar la influencia sobre el grado de combustión de la tasa de inyección de $\mathrm{CP}$, de la temperatura del viento y de su contenido de oxígeno. En la tabla I se incluyen los resultados de los ensayos, de donde resulta lo siguiente:

$\mathrm{Al}$ incrementar el caudal de $\mathrm{CP}$ desde 5 hasta 25 $\mathrm{g} / \mathrm{min}$ (lo que corresponde a $50-250 \mathrm{~g} \mathrm{CP} / \mathrm{m}^{3}$ de viento o aproximadamente $50-250 \mathrm{~kg} \mathrm{CP} / \mathrm{t}$ a), dis-

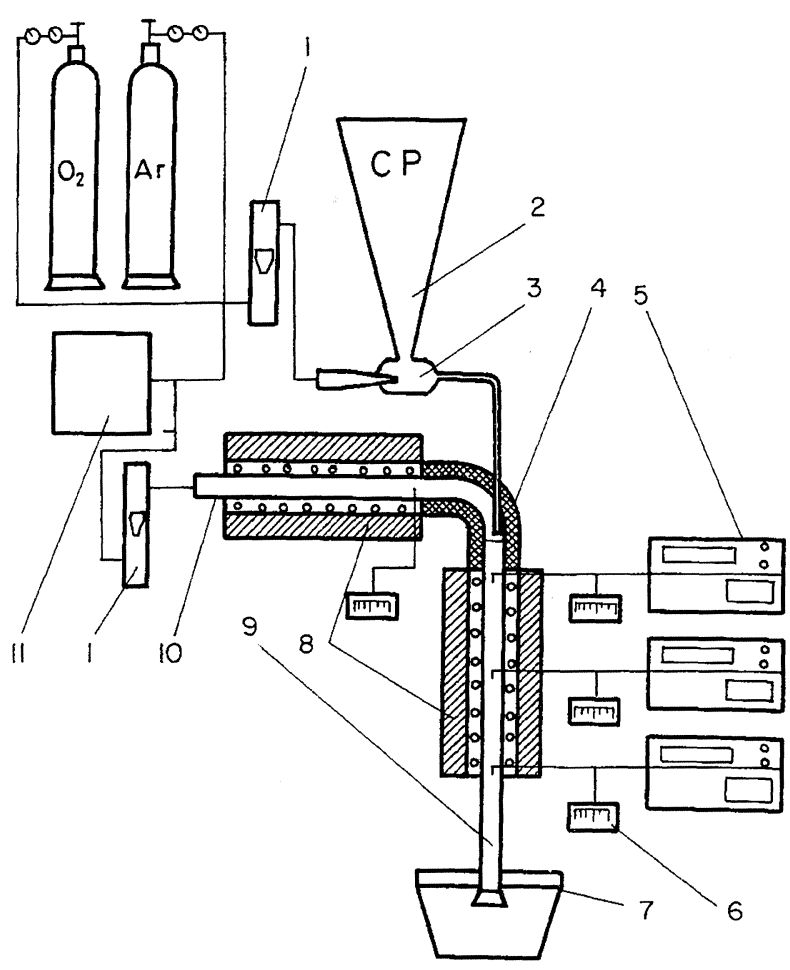

FIG. 1.- Esquema de la instalación de laboratorio: 1: Rotámetros, 2: Dosificador de CP, 3: Inyector, 4: Unión aislante, 5: Analizador de gases, 6: Sensores de temperatura, 7: Depósito de agua, 8: Horno eléctrico, 9: Cámara de combustión (tubo de cuarzo), 10: Tubo de cuarzo para precalentar el aire, 11: Soplante.

FIG. 1.- Scheme of laboratory rig: 1: Rotameters, 2: PC feeder, 3: Injector, 4: Insulating connection, 5: Gases analyser, 6: Temperature sensors, 7: Vessel with water, 8: Electrical furnaces, 9: Combustion chamber (quartz tube), 10: Quartz. tube for preheating air, 11: Air blower.
Tabla I.- Influencia de diferentes parámetros tecnológicos en el grado de combustión, $G$, del $\mathrm{CP}$

TABLE I.- Effect of different technological parameters on the $\mathrm{G}$ degree of PC combustion

\begin{tabular}{|l|c|c|}
\hline \multicolumn{1}{|c|}{ Parámetro } & Intervalo & $\Delta G, \%$ \\
\hline Temperatura del & $700-800$ & 3 \\
viento, ${ }^{\circ} \mathrm{C}$ & $800-900$ & 2,5 \\
& $900-1.000$ & 2 \\
& $1.000-1.100$ & 2 \\
\hline Contenido de $\mathrm{O}_{2}$ & $21-25$ & 12 \\
en el viento, \% & $25-30$ & 9 \\
& $30-35$ & 6 \\
& $35-40$ & 3,5 \\
\hline Caudal de $\mathrm{CP}$, & $5-10$ & -8 \\
g/min & $10-15$ & -8 \\
& $15-20$ & -9 \\
& $20-25$ & -10 \\
\hline
\end{tabular}

minuye su grado de combustión un $37 \%$ para otras condiciones iguales. El incremento de la temperatura del viento sólo aumenta un poco el grado de combustión del CP (2-3\% por cada $\left.100{ }^{\circ} \mathrm{C}\right)$. La concentración de oxígeno en el viento influye notablemente en el grado de combustión del CP. Un incremento del contenido de oxígeno en el viento de un $9 \%$ (desde 21 hasta $30 \%$ ), aumenta el grado de combustión del CP un $21 \%$; en este caso, se debe tener en cuenta que esta dependencia tiene un carácter no lineal.

La débil influencia de la temperatura del viento en el grado de combustión del CP, en comparación con la influencia de la concentración de oxígeno, confirma la conclusión sobre el carácter de difusión del proceso analizado.

En el CENIM se estudia el comportamiento frente a la combustión de diferentes carbones en una cámara de combustión, cuyo esquema se ofrece en la figura 2, que simula las condiciones en el raceway del horno alto (12-14). En la figura 3 se muestra la planta piloto en donde está instalada la cámara de combustión.

La tabla II ofrece la composición de carbones estudiados y la tabla III muestra los resultados de nueve ensayos realizados con los carbones. Los cinco primeros ensayos corresponden a dos carbones con un contenido alto de volátiles $(32 \%)$ y los cuatro últimos corresponden a un carbón con un contenido medio de volátiles (17\%).

Los rendimientos de la combustión del carbono al final del ensayo son próximos a los que se esperan en condiciones industriales, debido a que las temperaturas finales se aproximan a las que existen en el raceway. Se puede observar una tendencia hacia un grado de combustión más elevado para el 


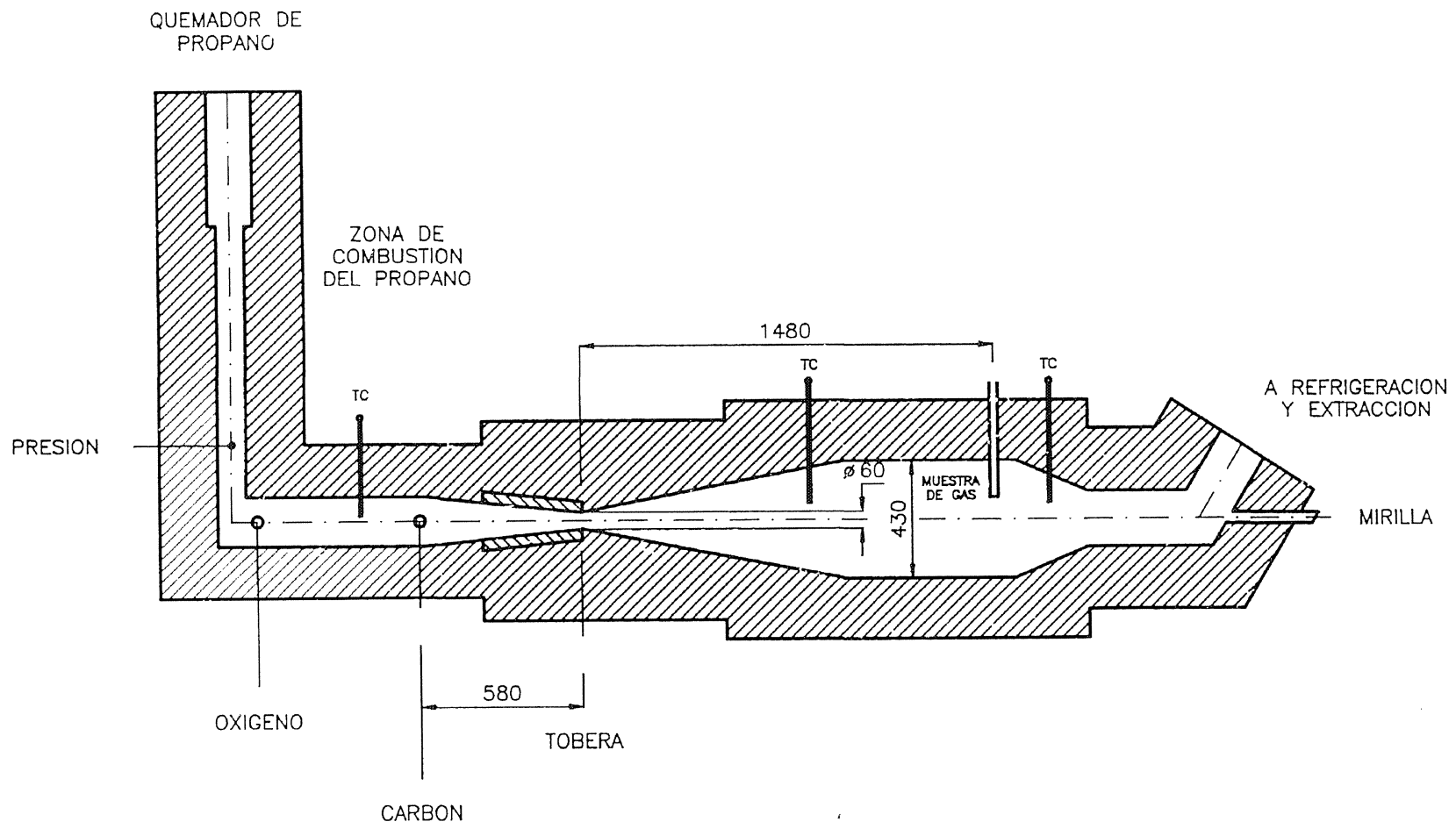

FIG. 2.- Esquema de la cámara de combustión del CENIM.

FIG. 2.- CENIM coal combustion device scheme.

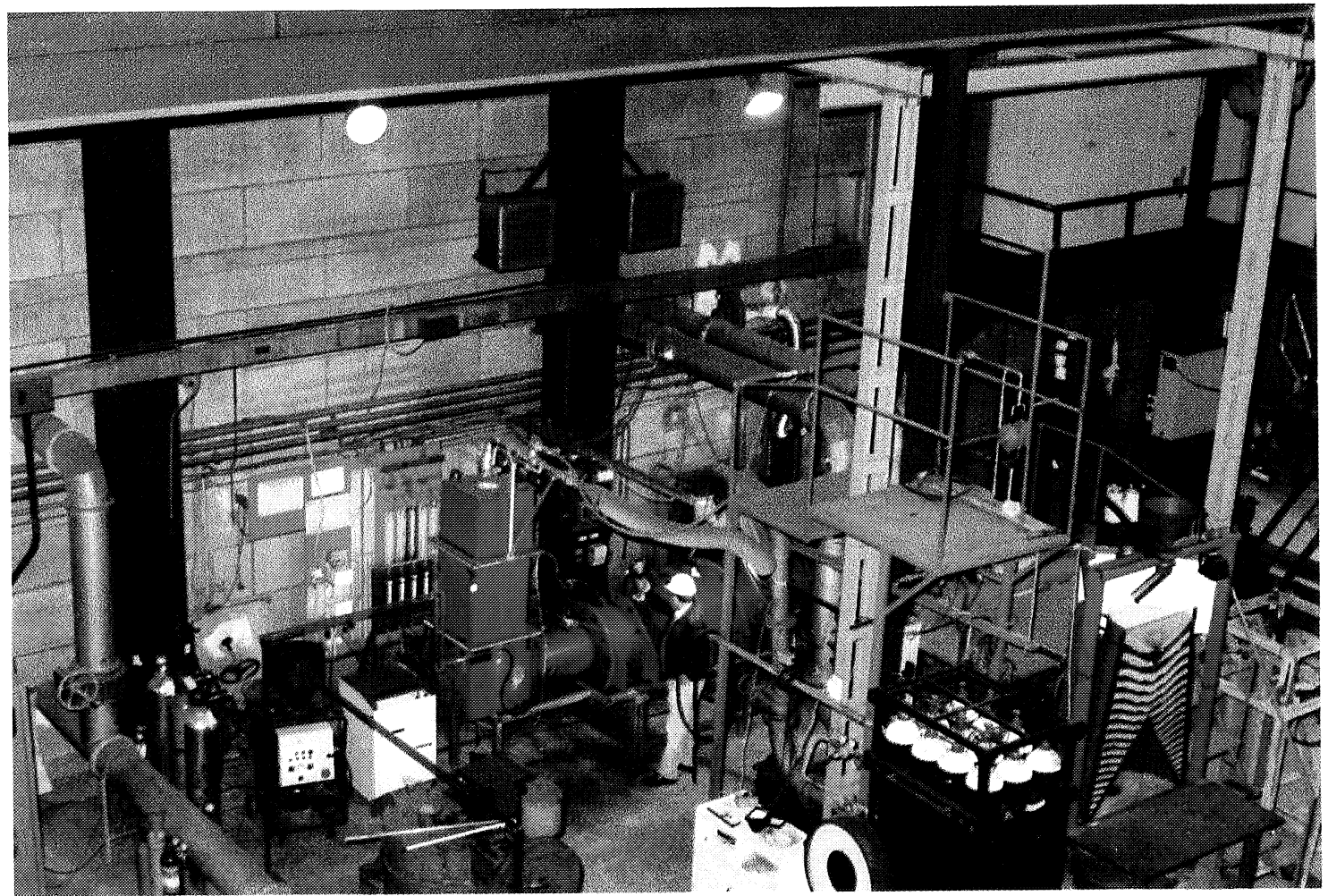

FIG. 3.- Vista de la planta piloto para ensayos de combustión del CENIM.

FIG. 3.- View of the CENIM pilot plant for combustion tests.

carbón con contenido medio de volátiles, lo que se puede explicar en relación con la producción de alquitrán (15).
En cualquier caso, la interpretación de los resultados es un asunto delicado: por ejemplo, parece que existe una tendencia hacia un mayor grado de 
TABLA II.- Análisis químico de carbones, \% en masa

TABLE II.-Chemical analysis of coals, wt \%

\begin{tabular}{|c|l|r|r|r|}
\hline \multicolumn{2}{|c|}{ Análisis } & \multicolumn{3}{|c|}{ Carbones } \\
\cline { 3 - 5 } Inmediato & $C_{1}$ & $C_{2}$ & \multicolumn{1}{|c|}{$C_{3}$} \\
\hline \multirow{5}{*}{ Elemental } & Humedad & 1,25 & 1,4 & 0,8 \\
& Ceniza & 7,08 & 7,3 & 7,7 \\
& Volátiles & 30,56 & 34,1 & 16,7 \\
& Carbono fijo & 61,11 & 58,6 & 75,6 \\
\hline \multirow{5}{*}{} & Carbono & 82,81 & 80,5 & 85,4 \\
& Hidrógeno & 4,90 & 5,1 & 3,9 \\
& Nitrógeno & 1,54 & 1,6 & 1,1 \\
& Azufre & 1,04 & 0,75 & 0,72 \\
\hline
\end{tabular}

combustión para flujos más elevados a la escala usada en estos ensayos (lo cual se puede explicar en términos de una concentración más elevada del calor generado), mientras que en las aplicaciones industriales puede ser crítica la mezcla del carbón inyectado y el viento caliente. Una tendencia que se puede apreciar y que puede ser de interés en aplicaciones reales es la influencia en la combustión de la relación entre el calor sensible del viento y el flujo de carbón. Esto se puede explicar en términos de un mayor desprendimiento de los volátiles a velocidades de calentamiento más elevadas, fenómeno descrito en varias ocasiones (16 y 17).

\section{MÉTODOS DE INTENSIFICACIÓN DE LA COMBUSTIÓN DEL CP}

El ser la difusión la etapa controlante en el proceso de combustión $\mathrm{CP}$ en la zona de toberas del horno alto, hecho demostrado de forma teórica y experimental, permite proponer las medidas siguientes para intensificar la combustión:
- Enriquecimiento del viento con oxígeno.

- Mejorar el mezclado del CP con el oxidante.

- Activación de los procesos de adsorción en la superficie de las partículas de carbono.

- Utilización de sustancias químicas-activadoras o de catalizadores de la combustión.

- Optimización de la molienda del carbón considerando su composición y su influencia sobre los parámetros del viento.

\subsection{Enriquecimiento del viento con oxígeno}

La mejora que se consigue al aumentar la concentración de oxígeno en el viento, cuando se inyecta $\mathrm{CP}$ en el horno alto, se ha demostrado en ensayos industriales (18 y 19).

A continuación, se analizan los resultados de los ensayos industriales realizados en el HA-1 (de $1.033 \mathrm{~m}^{3}, 7,2 \mathrm{~m}$ diámetro del crisol y 14 toberas) de la fábrica siderúrgica de Donetsk (Ucrania) con enriquecimiento del viento con oxígeno hasta un 25,3-26,0 \% (períodos I y II en la tabla IV). En el período base, el horno operó con un contenido de oxígeno en el viento del 22,5\%.

El aumento de la temperatura adiabática al enriquecer el viento con oxígeno se compensa con el incremento de los caudales de gas natural y CP.

Los resultados obtenidos (Tabla IV y Fig. 4) indican que la productividad del horno crece un 1,6-1,8 \% por cada unidad porcentual complementaria de oxígeno. El consumo de coque (recalculado) se reduce un $16,3 \%$, y el consumo de combustible un 2,7-4,8\%. El contenido de azufre en el arrabio experimenta un ligero descenso. Desde 1989 , los hornos de esta fábrica operan con viento que contiene un $24-26 \%$ de oxígeno.

TABLA III.- Resultado de los ensayos con carbón en la cámara de combustión del CENIM

TABLE III.- Results of the coals tests in the CENIM combustion chamber

\begin{tabular}{|c|c|c|c|c|c|c|c|}
\hline Carbón & $\begin{array}{c}\text { Flujo de } \\
\text { carbón, } \\
\mathrm{kg} / \mathrm{h}\end{array}$ & $\begin{array}{c}\mathrm{O}_{2} \text { en el } \\
\text { viento, } \\
\%\end{array}$ & $\mathrm{~kg} \mathrm{CP} / \mathrm{t}$ a & $\begin{array}{c}\text { Temp. del } \\
\text { viento, } \\
{ }^{\circ} \mathrm{C}\end{array}$ & $\begin{array}{c}\text { Grado de } \\
\text { combustión, } \\
\%\end{array}$ & $\begin{array}{c}\text { Temperat. } \\
\text { final, } \\
{ }^{\circ} \mathrm{C}\end{array}$ & $\begin{array}{c}\text { Duración de } \\
\text { inyección, } \\
\text { min }\end{array}$ \\
\hline $\mathrm{C}_{1}$ & 45,0 & 24,0 & 232 & 1.033 & 57,2 & 1.727 & 16 \\
$\mathrm{C}_{1}$ & 41,4 & 22,0 & 175 & 1.025 & 52,9 & 1.692 & 16 \\
$\mathrm{C}_{1}$ & 40,0 & 22,5 & 178 & 1.125 & 65,8 & 1.728 & 18 \\
$\mathrm{C}_{2}$ & 41,4 & 19,9 & 159 & 1.025 & 61,3 & 1.728 & 18 \\
$\mathrm{C}_{2}$ & 57,1 & 22,5 & 174 & 1.027 & 66,6 & 1.800 & 11 \\
$\mathrm{C}_{3}$ & 54,6 & 18,6 & 183 & 1.059 & 71,2 & 1.692 & 11 \\
$\mathrm{C}_{3}$ & 54,6 & 21,1 & 151 & 1.041 & 70,5 & 1.757 & 11 \\
$\mathrm{C}_{3}$ & 49,8 & 21,0 & 126 & 1.091 & 73,3 & 1.778 & 11 \\
$\mathrm{C}_{3}$ & 54,6 & 21,3 & 140 & 1.015 & 77,6 & 1.728 & 11 \\
\hline
\end{tabular}


TABLA IV.- Parámetros de operación del HA-1, de la planta siderúrgica de Donetsk, con viento enriquecido con oxígeno

TABLE IV.- Parameters of BF-I at Donetsk Steel Plant with oxygen enrichment of blast

\begin{tabular}{|c|c|c|c|c|}
\hline \multirow[b]{2}{*}{ Parámetros } & \multirow[b]{2}{*}{ Unidades } & \multicolumn{3}{|c|}{ Períodos } \\
\hline & & Base & I & II \\
\hline Duración del ensayo & día & 29 & 9 & 15 \\
\hline Consumo de materiales: & & & & \\
\hline - sínter & $\mathrm{kg} / \mathrm{t} \mathrm{a}$ & 839 & 820 & 676 \\
\hline - pelets & $\mathrm{kg} / \mathrm{t} \mathrm{a}$ & 577 & 697 & 651 \\
\hline - mineral de hierro & $\mathrm{kg} / \mathrm{t} \mathrm{a}$ & 33 & 72 & 68 \\
\hline - adiciones metálicas & $\mathrm{kg} / \mathrm{t} \mathrm{a}$ & 180 & 142 & 132 \\
\hline - caliza & $\mathrm{kg} / \mathrm{t} \mathrm{a}$ & 147 & 157 & 150 \\
\hline Viento: & & & & \\
\hline - caudal & $\mathrm{m}^{3} / \mathrm{min}$ & 2064 & 1923 & 1944 \\
\hline - exceso de presión & $\mathrm{kPa}$ & 226 & 210 & 231 \\
\hline - temperatura & ${ }^{\circ} \mathrm{C}$ & 1101 & 1093 & 1100 \\
\hline - contenido de $\mathrm{O}_{2}$ & $\%$ & 22,5 & 25,3 & 26,0 \\
\hline - caudal de $\mathrm{O}_{2}$ & $\mathrm{~m}^{3} / \mathrm{t} \mathrm{a}$ & 25,5 & 68,1 & 80,1 \\
\hline Caudal de gas natural & $\mathrm{m}^{3} / \mathrm{t} \mathrm{a}$ & 72,4 & 86,3 & 100,2 \\
\hline Caudal de CP & $\mathrm{kg} / \mathrm{t} \mathrm{a}$ & 72,0 & 85,7 & 76,9 \\
\hline Gas del tragante & & & & \\
\hline - exceso de presión & $\mathrm{kPa}$ & 122 & 1.05 & 125 \\
\hline - temperatura & ${ }^{\circ} \mathrm{C}$ & 326 & 278 & 279 \\
\hline - volumen & $\mathrm{m}^{3} / \mathrm{t} \mathrm{a}$ & 2270 & 1909 & 1910 \\
\hline Grado de utilización de $\mathrm{CO}$ & $\%$ & 38,5 & 39,4 & 40,1 \\
\hline Consumo de coque & $\mathrm{kg} / \mathrm{t} \mathrm{a}$ & 456 & 382 & 382 \\
\hline Consumo de combustible & $\mathrm{kg} / \mathrm{t} \mathrm{a}$ & 598 & 582 & 569 \\
\hline Productividad & $\mathrm{t} / 24 \mathrm{~h}$ & 1747 & 1845 & 1846 \\
\hline Grado de red. directa & $\%$ & 26,1 & 29,5 & 26,7 \\
\hline Temp. adiabática & ${ }^{\circ} \mathrm{C}$ & 1993 & 1997 & 1916 \\
\hline Intensidad de fusión & $\mathrm{t} / \mathrm{m}^{3} \cdot 24 \mathrm{~h}$ & 0,831 & 0,784 & 0,783 \\
\hline Composición del arrabio: & & & & \\
\hline $\mathrm{Si}$ & $\%$ & 0,75 & 0,74 & 0,77 \\
\hline $\mathrm{Mn}$ & $\%$ & 0,76 & 0,86 & 0,91 \\
\hline S & $\%$ & 0,037 & 0,033 & 0,030 \\
\hline Composición de la escoria: & & & & \\
\hline $\mathrm{MgO}$ & $\%$ & 4,0 & 4,9 & 4,4 \\
\hline $\mathrm{Al}_{2} \mathrm{O}_{3}$ & $\%$ & 8,5 & 7,6 & 7,0 \\
\hline $\mathrm{MnO}$ & $\%$ & 1,36 & 0,90 & 0,89 \\
\hline S & $\%$ & 1,88 & 1,82 & 1,82 \\
\hline $\mathrm{CaO} / \mathrm{SiO}_{2}$ & & 1,28 & 1,25 & 1,26 \\
\hline Volumen de escoria & $\mathrm{kg} / \mathrm{t} \mathrm{a}$ & 425 & 459 & 459 \\
\hline
\end{tabular}

\subsection{Mejora del mezclado del CP con el oxidante}

A pesar de la evidente mejora que supone enriquecer el viento con oxígeno, la efectividad de su utilización desde el punto de vista de la gasificación del CP es insuficiente, debido a que la construcción de los dispositivos de toberas no garantiza una mezcla uniforme del CP con la corriente del viento.

La mejora de la combustión del CP se puede alcanzar a partir de un aumento de la concentración local de oxidante en la región donde se encuentra el $\mathrm{CP}$, mediante una mezcla previa del $\mathrm{CP}$ con el oxí- geno antes de la salida de la mezcla oxígeno/carbón en el canal del dispositivo de toberas. Existen construcciones diferentes de los dispositivos de toberas basados en este principio; una de ellas se emplea en Thyssen Stahl (R.F.A.) (20).

Sin embargo, es más efectivo aumentar la concentración de oxígeno después del calentamiento y desprendimiento de las sustancias volátiles y del calentamiento del coque residual del carbón, ya que el aumento de la concentración de oxígeno sólo acelera la combustión del coque residual del $\mathrm{CP}$. Además, con una mezcla previa del $\mathrm{CP}$ con el oxígeno, se 


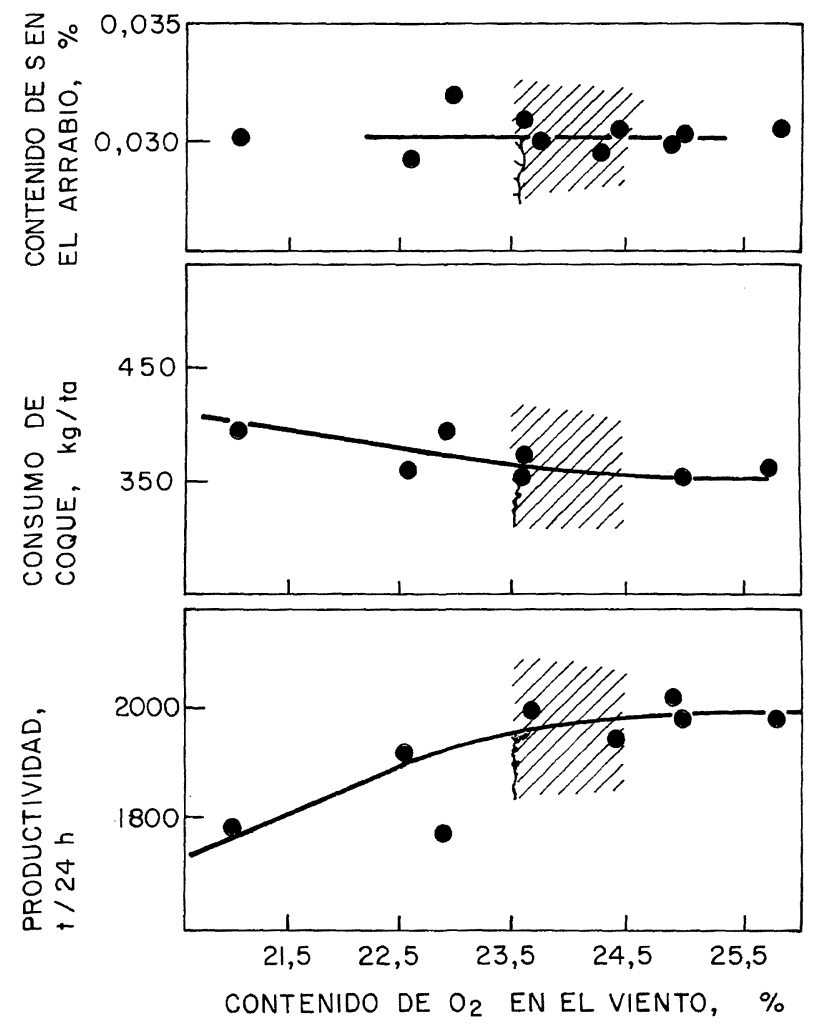

Fig. 4.- Influencia de la concentración de oxígeno del viento en la operación del horno alto.

FIG. 4.- Effect of oxygen concentration in the blast on blast furnace operation parameters.

queman inicialmente las fracciones finas y, por consiguiente, su combustión se producirá en condiciones más favorables. La combustión de las partículas más gruesas, que se produce posteriormente, ocurre en una zona con menor concentración de oxígeno. Naturalmente, para poner en práctica la propuesta es necesario disponer de una lanza individual para introducir el oxígeno en el dispositivo de toberas.

Para garantizar el impacto oportuno del oxígeno en la corriente de $\mathrm{CP}$ precalentado, es necesario calcular la profundidad de su penetración y la trayectoria de desplazamiento en el dispositivo de toberas mediante las leyes de la Hidráulica y de la Aerodinámica del chorro en la corriente portadora. La profundidad de penetración del chorro en la corriente se determina mediante la ecuación

$$
h=k d \frac{V 2}{V 1} \sqrt{\frac{\rho_{2}}{\rho_{1}}} \operatorname{sen} \alpha
$$

donde

$h=$ Profundidad de penetración del chorro, $\mathrm{m}$;

$k=$ Coeficiente, que depende del ángulo de impacto y de la estructura del chorro (para un chorro redondo de $90^{\circ}, k=2,2$ );

$d=$ Diámetro del chorro en la boca de la tobera, $\mathrm{m}$;
$V_{1}, V_{2}=$ Velocidad de la corriente portadora y del chorro, $\mathrm{m} / \mathrm{s}$;

$\rho_{1}, \rho_{2}=$ Densidad de la corriente portadora y del chorro, $\mathrm{kg} / \mathrm{m}^{3}$;

$\alpha=$ Ángulo de ataque del chorro, en grados.

A partir de los resultados de los cálculos, se elaboró la construcción del dispositivo de toberas para la adición individual de oxígeno. Los parámetros seleccionados para la lanza de inyección y para el oxígeno garantizan la profundidad de su penetración, excluyendo la posibilidad de que el chorro de oxígeno queme el cuerpo de la tobera. Además, el chorro de oxígeno debe separar las partículas de carbón de las paredes del canal interno de la tobera y proteger los elementos del dispositivo de toberas de su desgaste por abrasión, lo que supone un aumento de su duración. Para realizar las investigaciones en el horno alto se construyó una instalación piloto (Fig. 5) similar a la descrita en la referencia (21).

La efectividad del método utilizado para la adición del oxígeno se determina mediante la toma de muestras de materiales en el dispositivo de toberas y en el crisol, empleando una sonda refrigerada por agua.

Los resultados del análisis mineralógico de las muestras tomadas indican que, con la adición individual de oxígeno, la cantidad de partículas de $\mathrm{CP}$ inquemadas en el borde de la tobera es un 15-20\% menor que en la tecnología usualmente empleada para el enriquecimiento del viento (Fig. 6). En los ensayos con adición local de oxígeno, se observa que a la distancia de $0,5 \mathrm{~m}$ del borde de la tobera la cantidad de partículas de CP inquemadas también es menor.

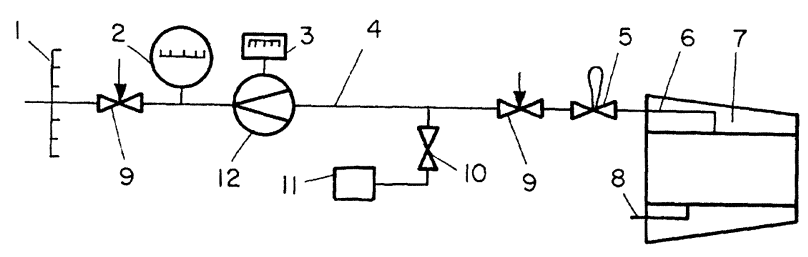

FIG. 5.- Esquema de la instalación industrial experimental para el suministro de oxígeno por tobera: 1: Colector de oxígeno, 2: Manómetro, 3: Medidor de caudal de oxígeno, 4: Conducto de viento, 5: Válvula inversa, 6: Tubuladura para oxígeno, 7: Tobera, 8: Conducto de CP, 9: Válvula de bola, 10: Válvula, 11: Válvula de seguridad, 12: Diafragma.

FIG. 5.- Scheme of an experimental industrial installation for oxygen delivery by tuyere: 1: Oxygen collector, 2: Manometer, 3: Oxygen flow gauge, 4: Oxygen conduct, 5: Invers valve, 6: Oxygen tubulature, 7: Tuyere, 8: CP conduct, 9: Ball valve, 10: Valve, 11: Security valve, 12: Diaphragm. 


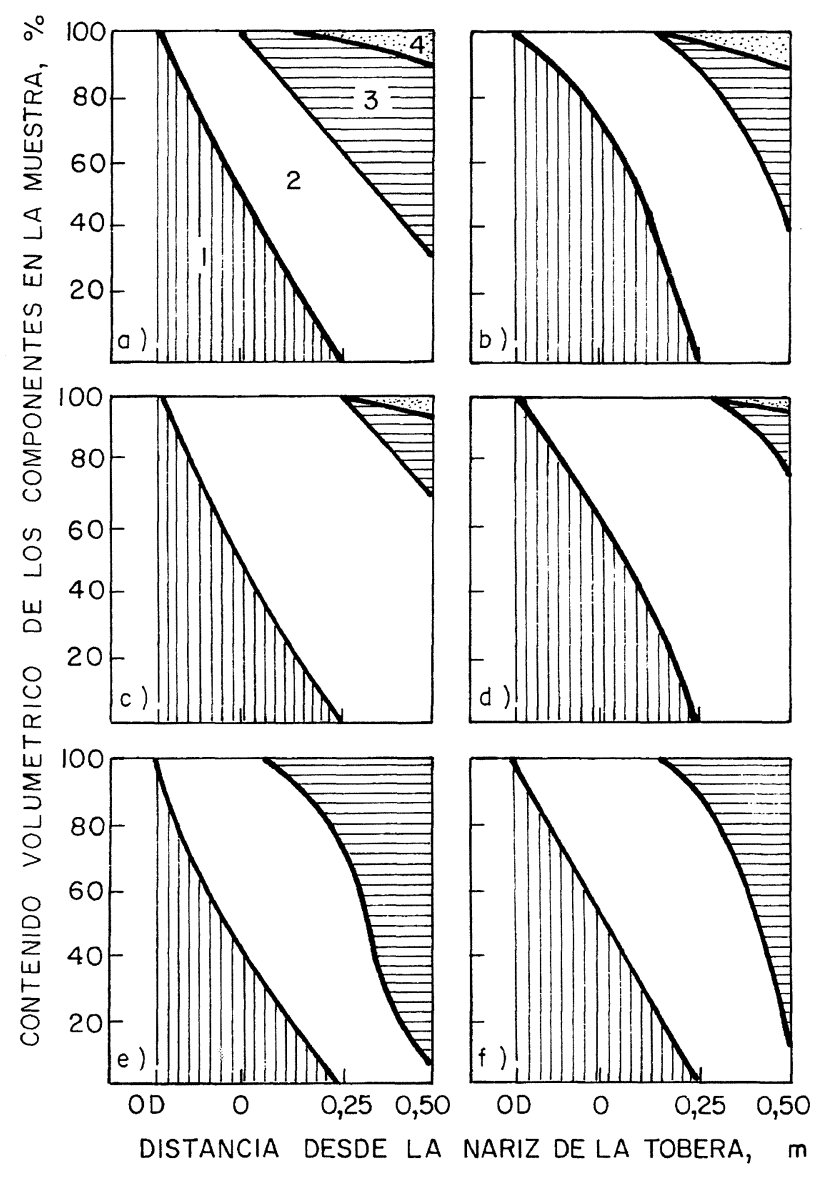

FIG. 6.- Efecto del suministro individual de oxígeno sobre la combustión del $\mathrm{CP}$ en la tobera y raceway. (a), (c) y (e) ensayos con suministro de oxígeno; (b), (d) y (f) ensayos sin suministro de oxígeno. 1: CP (sin alterar), 2: CP residual (char), 3: Escoria, 4: Coque, OD: Punto de suministro de oxígeno.

FIG. 6.- Effect of individual oxygen delivery on PC combustion in the tuyere and raceway. (a), (c) y (e) experiments with oxygen delivery; $(b),(d)$ y $(f)$ experiments without oxygen delivery.

1: PC (unchanged), 2: PC residues (rings), 3: Slag, 4: Coke, OD: Oxygen delivery point.

Los datos obtenidos confirman la intensificación de la combustión del CP al realizar la adición individualizada de oxígeno.

Actualmente, en la fábrica siderúrgica de Donetsk (Ucrania) se ha construido un sistema individualizado para la adición de oxígeno.

\subsection{Empleo de procedimientos físicos}

En la actualidad, se desarrollan trabajos para la intensificación de la combustión de los combustibles mediante la aplicación de campos eléctricos y magnéticos.

Para electrificar el CP inyectado en el crisol del horno alto, se puede emplear el procedimiento pro- puesto en (21), que consiste en lo siguiente: Las partículas de CP durante la preparación y transporte neumático desde la zona de molienda hasta el horno alto adsorben en su superficie moléculas de gases, principalmente nitrógeno y oxígeno. El mecanismo de adsorción consiste en que las moléculas gaseosas, en la interfase carbón-gas, quedan retenidas sobre la superficie de las partículas de carbón por fuerzas de atracción de Van der Waals (adsorción física). Como resultado se produce un recubrimiento completo de la superficie de las partículas de las moléculas de carbón por las moléculas de los gases, dando lugar a una capa de adsorción.

$\mathrm{Al}$ entrar el $\mathrm{CP}$ en el campo eléctrico, se polarizan las moléculas adsorbidas de oxígeno, lo que provoca una consolidación más fuerte de éstas en la superficie de las partículas de carbón, y la adsorción física se convierte en una absorción química, acelerando la reacción de interacción del carbono de las partículas de CP con el oxígeno en el período de combustión. La quimisorción del oxígeno ocurrida provoca la desorción de las moléculas de nitrógeno y de otros gases desde la superficie de las partículas de carbón.

El desarrollo de estos procesos se explica por el menor valor de la energía de polarización del oxígeno, y también por la energía de formación de las moléculas de oxígeno $\left(-\Delta H^{\circ}=249,36 \mathrm{~kJ} / \mathrm{mol}\right)$, que es menor que la energía de formación de las moléculas de nitrógeno $\left(-\Delta H^{\mathrm{o}}=773,03 \mathrm{~kJ} / \mathrm{mol}\right)$.

El campo eléctrico disminuye la energía de salida del electrón desde las partículas de carbón, y su salida facilita la polarización del oxígeno y el paso de la adsorción física del oxígeno a la química.

Por consiguiente, la intensificación de la combustión del $\mathrm{CP}$ tratado mediante campos eléctricos se alcanza a partir de la disociación de las moléculas de oxígeno adsorbidas en iones y, fundamentalmente, de la activación de las partículas de carbón por su preparación para la reacción de combustión con el oxígeno, como resultado de la desorción de los átomos y moléculas de nitrógeno.

En la figura 1 se representa el equipo de laboratorio que también se ha utilizado para realizar los ensayos de preparación del CP empleando campos eléctricos. En estos ensayos se demuestra la posibilidad de aumentar el grado de combustión del CP en un $20-25 \%$ (Fig. 7).

El dispositivo para la electrificación del CP se utilizó en una tobera de un horno alto en operación. Los resultados de los ensayos confirman las posibilidades técnicas de realización del procedimiento para intensificar la combustión del CP.

\subsection{Uso de aditivos químicos}

La combustión del CP se puede intensificar mediante el empleo de sustancias activadoras, 


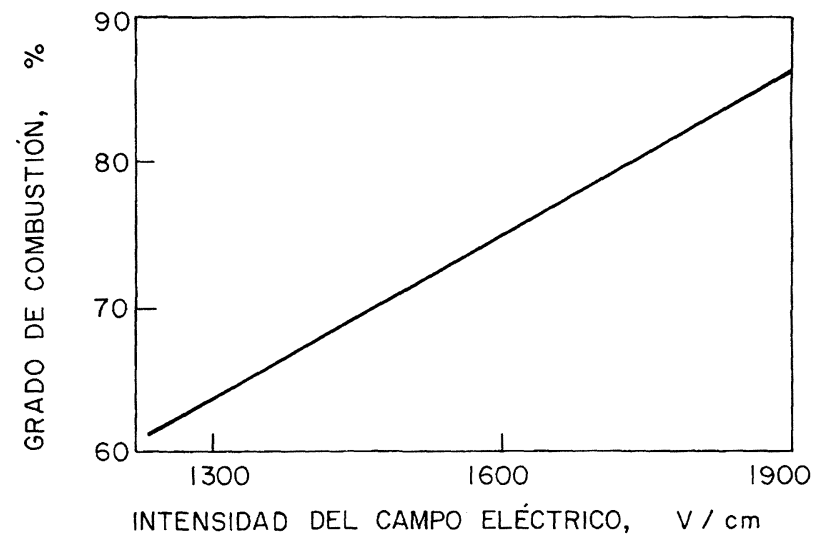

FIG. 7.- Dependencia entre el grado de combustión del CP y la intensidad del campo eléctrico.

FIG. 7.- Dependence between the degree of PC combustion on the magnitude of the electrical field.

mezcladas con el $\mathrm{CP}$, que aumentan la concentración local de oxidantes $\left(\mathrm{O}_{2}\right.$ y $\left.\mathrm{CO}_{2}\right)$ en la zona de inicio de la combustión, lo que acelera la gasificación del CP. Estas sustancias pueden ser nitratos de metales alcalinos, alcalino-térreos, carbonatos y otras.

Se debe destacar que la inyección en el crisol de aditivos, individual o en mezcla con el $\mathrm{CP}$, también se puede emplear para otros fines, tales como:

- Mejorar la composición de la escoria y las condiciones de desulfuración del arrabio en el horno, mediante la inyección de fundentes y otros componentes que incrementen la actividad de la escoria.

- Evitar los "emborricamientos" del crisol por masas viscosas de los materiales de la carga mediante la inyección de sustancias que disminuyan la viscosidad de la escoria.

- Reciclado de residuos industriales que contienen hierro.

- Disminución del contenido de silicio en el arrabio mediante la inyección de óxidos de hierro o de manganeso.

En la tabla V se incluyen los resultados de algunos estudios termográficos realizados para determinar la influencia de algunos activadores de la combustión y de materiales que contienen hierro, en las zonas de temperaturas de combustión de las mezclas combustibles (22 y 23). Su análisis manifiesta que al adicionar al $\mathrm{CP}$ un 5-10\% de nitratos de sodio o de potasio o un $5 \% \mathrm{FeO}$, se reduce considerablemente la temperatura de ignición de las partículas de CP y la combustión se realiza a más bajas temperaturas. Los finos de sínter y de pelets intensifican la combustión del $\mathrm{CP}$ cuando sus proporción en la mezcla es más elevada (entre el 20 y $30 \%$ ).
TABla V.- Características de las temperaturas de combustión de mezclas de CP y materiales no combustibles

TABLE V.- Temperature characteristics of PC mixtures burning

\begin{tabular}{|l|c|c|c|}
\hline \multirow{2}{*}{$\begin{array}{c}\text { Composición de } \\
\text { mezclas }\end{array}$} & \multicolumn{3}{|c|}{ Temperatura, ${ }^{\circ} \mathrm{C}$} \\
\cline { 2 - 4 } & $\begin{array}{c}\text { Inicio de salida } \\
\text { de volátiles }\end{array}$ & Ignición & $\begin{array}{c}\text { Final del } \\
\text { proceso }\end{array}$ \\
\hline $\mathrm{CP}$ (sin adiciones $)$ & 360 & 570 & 1.320 \\
$\mathrm{CP}+5 \% \mathrm{FeO}$ & 320 & 585 & 1.340 \\
$\mathrm{CP}+10 \% \mathrm{FeO}$ & 320 & 560 & 1.340 \\
$\mathrm{CP}+20 \% \mathrm{FeO}$ & 320 & 580 & 1.280 \\
$\mathrm{CP}+30 \% \mathrm{FeO}$ & 310 & 580 & 1.280 \\
$\mathrm{CP}+10 \%$ sínter & 340 & 605 & 1.280 \\
$\mathrm{CP}+20 \%$ sínter & 300 & 500 & 1.340 \\
$\mathrm{CP}+30 \%$ sínter & 270 & 450 & 1.380 \\
$\mathrm{CP}+10 \%$ pelets & 325 & 595 & 1.325 \\
$\mathrm{CP}+20 \%$ pelets & 300 & 500 & 1.300 \\
$\mathrm{CP}+30 \%$ pelets & 355 & 580 & 1.200 \\
$\mathrm{CP}+10 \%$ lodos & 365 & 620 & 1.400 \\
$\mathrm{CP}+20 \%$ lodos & 325 & 600 & 1.300 \\
$\mathrm{CP}+30 \%$ lodos & 320 & 580 & 1.275 \\
$\mathrm{CP}+5 \% \mathrm{NaNO}_{3}$ & 320 & 480 & 1.290 \\
$\mathrm{CP}+10 \% \mathrm{NaNO}_{3}$ & 340 & 525 & 1.140 \\
$\mathrm{CP}+5 \% \mathrm{KNO}_{3}$ & 320 & 540 & n.d. \\
$\mathrm{CP}+10 \% \mathrm{KNO}_{3}$ & 340 & 560 & 1.170 \\
\hline
\end{tabular}

La adición de lodos de depuración no influye prácticamente en los parámetros analizados. Por tanto, la inyección de esta mezcla puede ser útil desde el punto de vista del reciclado de los residuos y de una menor contaminación del medio ambiente.

En la fábrica siderúrgica de Donetsk se realizan ensayos industriales con inyección de mezcla de CP y diferentes fundentes, diluyentes de escoria e intensificadores de la combustión (24).

Las mezclas combustibles se preparan en el parque de carbones de las instalaciones de preparación e inyección del CP. En calidad de aditivo, al concentrado de hullas magras se le añaden dolomía en trozos, cal y espato flúor. Con el carbón y las adiciones correspondientes se formaron parvas individuales, que se cargan en las tolvas receptoras de carbón crudo del propio bloque. El posterior método de preparación de la mezcla combustible no se diferencia del método tecnológico convencional de preparación del CP.

En la tabla VI se analiza la efectividad de la utilización de las mezclas combustibles durante un período de casi cuatro meses operando con inyección de una mezcla de CP y dolomía. El análisis de los resultados muestra que, en comparación con el período previo a los ensayos, sin adición de dolomía, para iguales condiciones, el consumo de coque se reduce un $1,8 \%$ con un incremento de productividad del horno del $2 \%$.

La disminución de los consumos de coque y de combustible se supone que se produce por mejora del descenso de los materiales y de la distribución de 
TABLA VI.- Parámetros de operación del HA-1 con inyección de CP y dolomía

TABLE VI.- Parameters of BF-1 operation with injection of $P C$ and dolomite mixture

\begin{tabular}{|c|c|c|c|}
\hline \multirow{2}{*}{ Parámetros } & \multirow{2}{*}{ Unidades } & \multicolumn{2}{|c|}{ Períodos } \\
\hline & & I & II \\
\hline Productividad & $t / 24$ & 1735 & 1769 \\
\hline Intensidad de fusión & $\mathrm{t} / \mathrm{m}^{3} \cdot 24 \mathrm{~h}$ & 0,77 & 0,75 \\
\hline Duración del ensayo & día & 14 & 26 \\
\hline Caudal de mezcla inyectada & $\mathrm{kg} / \mathrm{t} \mathrm{a}$ & 77 & 69 \\
\hline Relación CP/dolomía & $\%$ & $100 / 0$ & $91 / 9$ \\
\hline Consumo de materiales: & & & \\
\hline - coque & $\mathrm{kg} / \mathrm{t} \mathrm{a}$ & 419 & 411 \\
\hline - sínter & $\mathrm{kg} / \mathrm{t} \mathrm{a}$ & 635 & 767 \\
\hline - pelets & $\mathrm{kg} / \mathrm{t} \mathrm{a}$ & 794 & 625 \\
\hline - mineral de hierro & $\mathrm{kg} / \mathrm{t} \mathrm{a}$ & 61 & 70 \\
\hline - adiciones metálicas & $\mathrm{kg} / \mathrm{t} \mathrm{a}$ & 210 & 235 \\
\hline - caliza & $\mathrm{kg} / \mathrm{t} \mathrm{a}$ & 186 & 190 \\
\hline Viento: & & & \\
\hline - caudal & $\mathrm{m}^{3} / \mathrm{t} \mathrm{a}$ & 1942 & 2049 \\
\hline - presión & $\mathrm{kPa}$ & 234 & 233 \\
\hline - temperatura & ${ }^{\circ} \mathrm{C}$ & 1095 & 1102 \\
\hline Gas del tragante: & & & \\
\hline - presión & $\mathrm{kPa}$ & 133 & 131 \\
\hline - temperatura & ${ }^{\circ} \mathrm{C}$ & 306 & 301 \\
\hline Grado de utilización de $\mathrm{CO}$ & $\%$ & 37 & 39 \\
\hline Composición del arrabio: & & & \\
\hline $\mathrm{Si}$ & $\%$ & 0,87 & 0,82 \\
\hline $\mathrm{Mn}$ & $\%$ & 0,75 & 0,92 \\
\hline$S$ & $\%$ & 0,027 & 0,031 \\
\hline Composición de la escoria: & & & \\
\hline $\mathrm{MgO}$ & $\%$ & 3,9 & 4,4 \\
\hline $\mathrm{Al}_{2} \mathrm{O}_{3}$ & $\%$ & 8,4 & 8,3 \\
\hline $\mathrm{MnO}$ & $\%$ & 0,80 & 0,84 \\
\hline $\mathrm{S}$ & $\%$ & 1,95 & 1,83 \\
\hline $\mathrm{CaO} / \mathrm{SiO}_{2}$ & & 1,23 & 1,26 \\
\hline Volumen de escoria & $\mathrm{kg} / \mathrm{t} \mathrm{a}$ & 494 & 465 \\
\hline Grado de reducción directa & $\%$ & 35,9 & 34,9 \\
\hline Temperatura adiabática & ${ }^{\circ} \mathrm{C}$ & 1974 & 1984 \\
\hline Paradas del horno & $\%$ & 1,6 & 0,9 \\
\hline
\end{tabular}

I - Antes de los ensayos; II - Período experimental.

la corriente gaseosa en el horno. Esto también se confirma en el período experimental por el aumento del grado de utilización del $\mathrm{CO}\left(\eta_{\mathrm{co}}\right)$ desde el 37 al $39 \%$, por la disminución del grado de reducción directa y por la disminución de las paradas del horno. La calidad del arrabio no varía prácticamente.

\subsection{Optimización de la molienda del carbón}

La necesidad de lograr un alto grado de gasificación de las partículas de $\mathrm{CP}$ en la zona de toberas requiere una molienda fina del carbón. Sin embargo, por dicho motivo, aumenta considerablemente el gasto de energía en la trituración, disminuye la productividad de los molinos y empeoran las condiciones de transporte neumático del $\mathrm{CP}$.

La economía de utilización del $\mathrm{CP}$ es más alta cuando la suma de los gastos en la preparación y combustión del CP es mínima. Esta magnitud, en
PTA/t carbón, puede expresarse de la siguiente forma:

$$
G_{\mathrm{t}}=G_{\mathrm{p}}+G_{\mathrm{m}}+G_{\mathrm{c}}
$$

donde

$G_{\mathrm{t}}=$ Gasto total, PTA/t carbón;

$G_{\mathrm{p}}=$ Gastos, con el consumo de energía en la preparación del carbón, PTA/t carbón;

$G_{\mathrm{m}}=$ Gastos, con las pérdidas de metal durante la elaboración del CP (desgaste de los equipos) y otros gastos de servicio, PTA/t carbón;

$G_{\mathrm{c}}=$ Gastos, con las pérdidas de combustible durante la combustión, PTA/t carbón.

La relación de estos gastos frente a la superficie específica del CP se representa en la figura 8. El tamaño de molienda óptimo se localiza en la zona sombreada.

Para comprobar el grado óptimo de molienda del $\mathrm{CP}$, se realizaron diferentes investigaciones con un concentrado de hullas magras (25).

La tendencia a reducir el tamaño de las partículas de carbón se explica por el deseo de aumentar la superficie de reacción de las partículas y así acelerar su gasificación. Sin embargo, la superficie específica de las partículas también aumenta por el choque térmico a alta temperatura, al impactar éstas en el chorro de viento caliente.

El resultado del análisis cromatográfico (Tabla VII) muestra que la superficie específica del CP de la fracción 50-63 $\mu \mathrm{m}$ es superior en sólo un 8,5\% a la superficie específica de la fracción 63-100 $\mu \mathrm{m}$. Después del choque térmico (a $1.000{ }^{\circ} \mathrm{C}$ ), esta diferencia se reduce al 3,3\%. Al pasar desde la

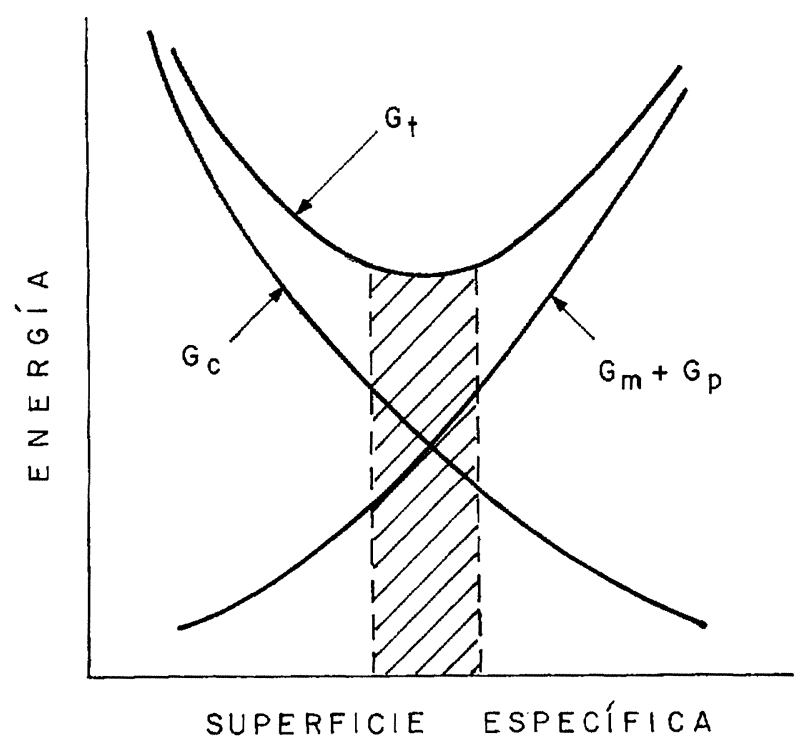

FIG. 8.- Principios de optimización de la molienda del carbón.

FIG. 8.-Principle of optimizing of coal grinding. 
TABla VII.- Variación de la superficie del CP durante el choque térmico $\left(1.000^{\circ} \mathrm{C}\right)$

TABLE VII.- Change in the CP surface during the thermal shock $\left(1,000{ }^{\circ} \mathrm{C}\right)$

\begin{tabular}{|c|c|c|c|}
\hline $\begin{array}{c}\text { Fracciones, } \\
\mu \mathrm{m}\end{array}$ & \multicolumn{3}{|c|}{$\begin{array}{c}\text { Superficie específica } \\
(S), \mathrm{m}^{2} / \mathrm{g}\end{array}$} \\
\cline { 2 - 4 } & $\mathrm{A}$ & $\mathrm{B}$ & $\Delta \mathrm{S}, \%$ \\
\hline$<50$ & 2,73 & 5,27 & 93 \\
$50-63$ & 2,17 & 4,98 & 129 \\
$63-100$ & 2,00 & 4,82 & 141 \\
$>100$ & 1,34 & 4,51 & 237 \\
Todo uno & 3,87 & 6,09 & 57 \\
\hline
\end{tabular}

A - Inicial; B - Después del choque térmico.

fracción 50-63 $\mu \mathrm{m}$ a la más fina $(<50 \mu \mathrm{m})$, el aumento de la superficie específica después del choque térmico es del $5,8 \%$.

Por tanto, el crecimiento de la superficie específica a partir del sucesivo paso a fracciones más finas es poco significativo en comparación con la influencia del choque térmico.

La cinética de combustión del CP se estudió con un derivatógrafo; la tabla VIII muestra los resultados de este estudio, que confirman que la finura de molienda del carbón varía sus propiedades físicoquímicas y las características de la combustión de la forma siguiente: aumenta la superficie, pero para las fracciones finas $(<50 \mu \mathrm{m})$ este crecimiento se reduce (ver el aumento de masa por la absorción química). El tiempo total de combustión de la fracción "todo uno" es igual al tiempo de combustión de la fracción más fina.

Por tanto, existe una molienda óptima para los carbones que se inyectan al horno alto. En las condi- ciones estudiadas, este valor se corresponde con un 60-80 \% de partículas con una granulometría comprendida entre 50 y $100 \mu \mathrm{m}$. La molienda del carbón $\mathrm{a}<50 \mu \mathrm{m}$ no está fundamentada tecnológicamente.

\section{DISTRIBUCIÓN DEL CP Y DEL VIENTO EN LA PERIFERIA DEL HORNO}

Las desviaciones que normalmente se producen en el caudal de viento soplado por toberas son del orden de $\pm 30-60 \%$. Estas variaciones se producen por una distribución heterogénea del viento a lo largo de la circular (anillo de viento) en los dispositivos de toberas y a las variaciones de permeabilidad de la carga al paso de los gases, en las diferentes zonas de toberas.

Para regular el caudal de viento caliente por toberas, se han diseñado válvulas de estrangulación y de compuertas especiales, así como salientes en la circular del viento (26). Las principales desventajas de estos procedimientos son las condiciones complejas de utilización de las válvulas a las altas temperaturas del viento (enriquecido con oxígeno), y también la disminución de la intensidad de la fusión como resultado de la reducción del caudal de viento por cerrarse (estrangularse) una parte de la sección de la conductora de viento caliente o de los portavientos del horno.

Debido a las irregularidades existentes en los hornos en el suministro del viento y para estabilizar el régimen de temperatura-viento, en la periferia del horno es necesario realizar un control racional de la distribución de los combustibles auxiliares por toberas.

Los sistemas modernos de inyección de combustibles, y en particular del $\mathrm{CP}$, garantizan la

TABLA VIII.- Parámetros del proceso de combustión del CP

TABLE VIII.- The parameters of PC burning process

\begin{tabular}{|l|c|c|c|c|c|}
\hline \multirow{2}{*}{ Parámetros } & \multicolumn{5}{|c|}{ Composición granulométrica, $\mu \mathrm{m}$} \\
\cline { 2 - 5 } & $<50$ & $50-63$ & $63-100$ & $>100$ & Todo uno \\
\hline Incremento de masa en la quimisorción, mg & 11 & 12 & 8 & 4 & 14 \\
Tiempo total, min & 99,5 & 105,5 & 100,0 & 111,0 & 99,5 \\
Tiempos de cada etapa, \%: & & & & & \\
- eliminación de humedad & 6,0 & 5,0 & 5,0 & 5,0 & 6,0 \\
- quimisorción & 8,0 & 7,7 & 5,5 & 6,5 & 7,0 \\
- eliminación de volátiles & 26,8 & 21,3 & 20,0 & 16,5 & 27,5 \\
- combustión de carbono residual & 59,2 & 66,0 & 69,5 & 72,0 & 59,5 \\
Temperaturas, ${ }^{\circ}$ C: & & & & & \\
- inicio de quimisorción & 100 & 102 & 110 & 110 & 119 \\
- inicio de desprendimiento de volátiles & 310 & 347 & 353 & 363 & 326 \\
- ignición & 551 & 563 & 568 & 565 & 557 \\
- máxima & 875 & 927 & 932 & 934 & 896 \\
- final de la combustión & 882 & 898 & 902 & 914 & 884 \\
\hline
\end{tabular}


posibilidad de distribución con una desviación máxima de su caudal por tobera del $\pm 3-5 \%$.

Para crear condiciones de gasificación de los combustibles auxiliares similares en todas las toberas, se utiliza frecuentemente el principio de proporcionalidad de los caudales inyectados por tobera. Así, el sistema de inyección de CP, construido por Paul Würth \& Arbed, prevé la medida individual del flujo de CP en cada tobera y la regulación de su caudal según el del viento caliente que entra en la determinada tobera (27). Mediante un ordenador en cada tobera, se mantiene una relación igual CP/viento. En Japón y en Ucrania funcionan sistemas análogos.

Los procedimientos y sistemas reseñados garantizan condiciones iguales de combustión del CP, o de otros combustibles auxiliares, en todas las toberas. Sin embargo, en este caso los volúmenes de gases que se forman en el crisol serán diferentes.

Como ya se describió anteriormente, estos problemas pueden resolverse mediante la inyección individual de oxígeno en el dispositivo de toberas. En (28) se propone cerrar la adición de oxígeno en las toberas por las que no se inyecta el CP. En otro trabajo (21) se elaboraron procedimientos de distribución del oxígeno para suministros irregulares del viento por tobera.

\section{COMPENSACIÓN DE LAS PERTURBACIONES PRODUCIDAS POR LA INYECCIÓN DE CP EN EL HORNO ALTO}

$\mathrm{Al}$ inyectar $\mathrm{CP}$, particularmente en grandes caudales, se producen variaciones en los procesos de intercambio térmico, reducción, formación de escoria y gasodinámica, que es necesario compensar para conseguir una marcha estable del horno.

El desarrollo de los procesos de reducción en la cuba del horno depende del volumen de gases reductores, de la altura de la zona de reserva térmica, de la proporción de coque en la carga, de la intensidad de fusión y de otros parámetros. Todos ellos se pueden considerar como un parámetro universal: el tiempo de contacto del gas reductor con los óxidos de hierro en la zona de intercambio térmico moderado.

Los cálculos muestran que al inyectar $\mathrm{CP}$ en caudales de $200 \mathrm{~kg} / \mathrm{t}$ a, el tiempo de contacto aumenta en $0,12 \mathrm{~s}$ en esa zona. Como resultado, el grado de reducción disminuye en $2-4 \%$ por cada $100 \mathrm{~kg} \mathrm{CP} / \mathrm{t}$ a (7).

La influencia del $\mathrm{CP}$ en el régimen térmico del crisol y en la temperatura adiabática de combustión se puede valorar con la utilización del balance térmico de la parte inferior de la zona de intercambio térmico. Al realizar el análisis de este balance, se obtiene la ecuación de la temperatura adiabática de combustión, que es necesaria para conservar en el nivel inicial la composición y temperatura del arrabio al inyectar $\mathrm{CP}(7)$ :

$T_{1}=T_{2}+\left(1-0,7 \frac{r_{\mathrm{d}_{0}}-r_{\mathrm{d}_{1}}}{r_{\mathrm{d}_{0}}}\right) \frac{k_{0}}{k_{1}} \frac{V_{0}}{V_{1}}\left(T_{0}-T_{2}\right)$

donde

$T_{\mathrm{o}}$ y $T_{1}=$ Temperatura adiabática de combustión en las condiciones iniciales (sin inyección de $\mathrm{CP}$ ) y nuevas (con inyección de $\mathrm{CP}$ ), ${ }^{\circ} \mathrm{C}$;

$T_{2}=$ Temperatura en la zona de intercambio térmico moderado, ${ }^{\circ} \mathrm{C}$;

$r_{\mathrm{d}_{\mathrm{o}}}$ y $r_{\mathrm{d}_{1}}=$ Grado de reducción directa en las condiciones iniciales y nuevas, $\%$;

$V_{\mathrm{o}}$ y $V_{1}=$ Volumen de gases del crisol en las condiciones iniciales y nuevas, $\mathrm{m}^{3} / \mathrm{t}$ coque;

$k_{\mathrm{o}} \mathrm{y} k_{1}=$ Consumo de coque en las condiciones iniciales y nuevas, $\mathrm{kg} / \mathrm{t}$ a.

De la ecuación [5] resulta que las condiciones tecnológicas de fusión determinan la magnitud de la temperatura adiabática de combustión. Así, por ejemplo; la disminución del grado de reducción directa en $1 \%$ disminuye la magnitud $T_{1}$ en $15-25^{\circ} \mathrm{C}$.

La disminución de la temperatura adiabática de combustión (en $100-200{ }^{\circ} \mathrm{C}$ por cada $100 \mathrm{~kg} \mathrm{CP} / \mathrm{t}$ a), así como el aumento del volumen de gases del crisol, se pueden compensar mediante:

- Incremento de la temperatura del viento o de su concentración de oxígeno.

- Disminución de la humedad del viento o del caudal de gas natural (si se realiza la inyección simultánea con $\mathrm{CP}$ ).

En la tabla IX se incluyen los datos calculados de la operación del horno alto (21), que permiten valorar la efectividad de diferentes métodos de compensación. Los datos iniciales se tomaron para las condiciones de la fábrica siderúrgica de Donetsk. El análisis de los resultados expuestos confirma que el aumento de la temperatura del viento es el parámetro de compensación más efectivo.

\section{CONCLUSIONES}

- La inyección de CP en los hornos altos es la tecnología más avanzada de fabricación de arrabio desde el punto de vista económico y ecológico. La efectividad de esta tecnología, con bajo consumo de coque, para niveles de inyección de $\mathrm{CP}$ del orden de $200 \mathrm{~kg} / \mathrm{t}$ a, se puede lograr si se garantiza la gasificación completa del $\mathrm{CP}$ en la zona de toberas, la distribución uniforme por la periferia del horno y un conjunto de medidas que compensen las perturbaciones del régimen térmico de fusión. 
TABLA IX.- Efectividad de las medidas de compensación al inyectar CP

TABLE IX.-Effectiveness of compensating measures under PCI

\begin{tabular}{|c|c|c|c|c|c|}
\hline \multirow{2}{*}{ Parámetros } & \multirow{2}{*}{$\begin{array}{l}\text { Período base sin } \\
\text { inyección } \mathrm{CP}\end{array}$} & \multicolumn{4}{|c|}{ Variación de parámetros por cada $100 \mathrm{~kg} \mathrm{CP} / \mathrm{t} \mathrm{a}$} \\
\hline & & $T \mathrm{v}$ & $G N$ & $\mathrm{O}_{2}$ & $W$ \\
\hline Temperatura del viento $(T v),{ }^{\circ} \mathrm{C}$ & 1.100 & +250 & 0 & 0 & 0 \\
\hline Caudal de gas natural $(G N), \mathrm{m}^{3 / \mathrm{t}} \mathrm{a}$ & 80 & 0 & $-36,4$ & 0 & 0 \\
\hline Caudal de oxígeno $\left(\mathrm{O}_{2}\right), \mathrm{m}^{3 / \mathrm{t}}$ a & 23,0 & 0 & 0 & $+53,5$ & 0 . \\
\hline Humedad del viento $(W), \%$ & 1,0 & 0 & 0 & 0 & $-2,5$ \\
\hline Productividad del horno, $\%$ & 98,5 & $+10,8$ & $-0,8$ & $+7,1$ & $-4,5$ \\
\hline Consumo de coque, $\mathrm{kg} / \mathrm{t} \mathrm{a}$ & 440 & $-170,8$ & $-91,7$ & $-116,7$ & -125 \\
\hline Consumo de combustible, $\mathrm{kg} / \mathrm{t} \mathrm{a}$ & 610 & $-45,8$ & $-13,3$ & $-1,25$ & -75 \\
\hline Volumen de gases de crisol, $\mathrm{m}^{3} / \mathrm{t}$ a & 2.100 & $-166,7$ & $-41,7$ & $-203,3$ & $-25,0$ \\
\hline Grado de utilización de $\mathrm{CO}, \%$ & 35,5 & $+1,88$ & $+0,83$ & $+1,25$ & $+2,25$ \\
\hline Grado de reducción directa, $\%$ & 30,0 & $-3,6$ & 0 & $-3,6$ & 0 \\
\hline
\end{tabular}

- Los cálculos teóricos y los ensayos de laboratorio muestran el carácter de difusión como etapa controlante del proceso de la combustión del CP en la zona de toberas del horno alto. Esto permite elaborar procedimientos de intensificación de la combustión del CP.

- La experiencia práctica en el horno alto operando con inyección de CP, demuestra la mejora de los parámetros técnico-económicos de fusión, (aumento de la productividad, disminución del consumo de coque y de otros elementos de adición), al aumentar el contenido de oxígeno en el viento hasta un $25-26 \%$.

- Se han elaborado métodos de intensificación de la combustión del CP, tales como la electrificación de las partículas de carbón y la inyección de mezclas de CP con sustancias químicas activadoras.

- Para la combustión rápida y completa del CP, es conveniente la inyección de carbón con granulometría fina. Sin embargo, esta molienda requiere grandes gastos de energía y empeora las condiciones de transporte neumático del CP. Debido a ello, se han desarrollado recomendaciones para optimizar la molienda del carbón inyectado.

- Para garantizar condiciones iguales de gasificación del CP por la periferia del horno, se puede emplear un sistema que controle proporcionalmente el caudal de viento caliente y, en caso de inyección individual del oxígeno por tobera, es posible utilizar este mismo sistema para nivelar la cantidad de oxidante en las diferentes zonas de las toberas.

- Para compensar las perturbaciones producidas al inyectar $\mathrm{CP}$, se pueden emplear parámetros de compensación tales como: la temperatura del viento y su contenido de oxígeno, la humedad del viento y el caudal de gas natural. El aumento de la temperatura del viento es el parámetro de compensación más efectivo.

\section{Agradecimiento}

Los autores agradecen los apoyos económicos recibidos, al Ministerio de Educación y Ciencia (Ref. Sab95-0151), ECSC (CE) (Agreement No. 7210.AA/936), ECSC (CE) (Agreement $\mathrm{N}^{\circ}$. 7210.AA/938), y a la Factoría Siderúrgica de Donetsk (Ucrania) la realización de los ensayos industriales.

\section{REFERENCIAS}

(1) PAt. No. 6207 (R.U.), 1831.

(2) Gue, Yun-Zhi y Ltu-Cai. Rev. Metall.-Cah. Inf. Tech., 77 (10), 1980: 16-18.

(3) BRundy, J.L. J. Met., 16 (8), 1964: 637-643.

(4) Bell, S.A., Pugh, I.L. y Snyder, B.I. J. Met., 20 (4), 1968: 85-88.

(5) Yaroshevskil, S.L., Yarmall, A.A. y Suplin, L.Z. Stal, (9), 1976: 788-792.

(6) Antonov, A.A. y Yarmall, A.A. Utilización del carbón pulverizado y gas natural para la fabricación de arrabio. Tejnika. Kiev (Ucrania), 1974: 188 págs. En ruso.

(7) YaroshevSKil S.L. Fabricación de arrabio con empleo de carbón pulverizado. Metallurgiya. Moscú, 1988: 176 págs. En ruso.

(8) Masumoto, T., Fugimori, H., Watanabe, Y. y Taguchi, S. Proc. European Ironmaking Congress. Vol. 2. Aachen (R.F.A.), 1985: 1-18.

(9) Steiler, J.M., Dufour, A., Libralesso, J.M., Helleisen, M. y Harting, W. Rev. Metall.-Cah. Inf. Tech., 92 (1011), 1995: 1.208-1.223.

(10) Pomerantsev V.V. Fundamentos prácticos de la teoría de la combustión. Energoatomizdat. Leningrado, 1986: 312 págs. En ruso.

(11) Andronov, V.N., Babich, A.I. y Yaroshevskil, S.L. Steel URSS-Engl. Tr., 18 (5), 1988: 197-199. 
(12) Isidro, A., Formoso, A., Pis J.J., Ferreira, S., Rivas, J.M., GarCIA, L. y CoReS A. Proc. VIII Int. Conf. on Coal Sci. and Tech. Vol. 1. Elsevier Science. Oviedo (España), 1995: 551-554.

(13) Formoso, A., Isidro, A., Cores, A. y Ferreira, S. Further developments in blast furnace injection technology. ECSC Agreement No. 7210 AA/936. 1994.

(14) Babich, A.I., Garcia, L., Formoso, A., Mochon, J. y CoRes, A. Rev. Metal. Madrid, 32 (1996). En prensa.

(15) HutnY, W.P. y PRICE, J.P. Ironmaking Steelmaking, 16 (3), 1989: 165-167.

(16) Sмоот, L.D. Fossil Fuel Combustion. Ed. W. Bartok y A.F. Sarofin. John Wiley \& Sons. Nueva York (EE.UU.), 1991: 682

(17) Bortz, S. y Flament, G. Ironmaking Steelmaking, 10 (5), 1983: 222-229.

(18) Lanbao, Ch., Guangchuen, G. y Shuhan Ma. Iron Steel (China), 23 (11), 1988: 1-10.

(19) Yaroshevskit, S.L., Didevich, A.V. y Babich, A.I. Steel URSS-Engl. Tr., 19 (12), 1989: 513-515.

(20) Peters, K.-H., Mohnkern H. y Lüngen, H.B. Proc. 1st Int. Congr. on Sci. and Tech. of Ironmaking. ISIJ. Sendai (Japón), 1994: 493-499.
(21) Babich, A.I., Yaroshevskil, S.L. y Tereshenko, V.P. Intensificación de la utilización del carbón pulverizado en el horno alto. Tejnika. Kiev (Ucrania), 1993: 198 págs. En ruso.

(22) Babich, A.I. e Ivleva, T.A. Izv. Vuzov. Chernaya Metallurgiya, (1), 1992: 113-115.

(23) Babich, A.I. e Ivleva, T.A. Izv. Vuzov. Chernaya Metallurgiya, (3), 1995: 75-76.

(24) Yaroshevskit, S.L., Skladanovskit, E.N., Stepanov, V.V. y BabICH, A.I. Steel URSS-Engl. Tr., 17 (6), 1987: 254-257.

(25) Andronov, V.N., Babich, A.I. y YaroshevskiI, S.L. Steel URSS-Engl. Tr., 18 (5), 1988: 197-199.

(26) Hiraoka, H., Nakajima, R., Hattori, M., Lino, B., HotтA, H. e Ito, H. Proc. Mc Master University Symp. No. 19. Ed. by W.-K. Lu. Hamilton, Ontario (Canadá), 1991, 255-266.

(27) Koster, J. y Limpach, R. Ironmaking Steelmaking, 10 (3), 1983: 104-108.

(28) 'Campbell, D.A., Flierman, G., Malgarini, G. y Smith R.B. Ironmaking Steelmaking, 19 (2), 1992: 120-125. 